\title{
Encoding and the Durability of Episodic Memory: A Functional Magnetic Resonance Imaging Study
}

\author{
Melina R. Uncapher and Michael D. Rugg \\ Center for the Neurobiology of Learning and Memory and Department of Neurobiology and Behavior, University of California, Irvine, Irvine, California
} 92697-3800

\begin{abstract}
Memories vary in their durability even when encoding conditions apparently remain constant. We investigated whether, under these circumstances, memory durability is nonetheless associated with variation in the neural activity elicited during encoding. Event-related functional magnetic resonance imaging data were acquired while volunteers semantically classified visually presented words. Using the "remember/know" procedure, memory for one-half of the words was tested after $30 \mathrm{~min}$ and for the remaining half after $48 \mathrm{~h}$. In several regions, including left hippocampus and left dorsal inferior frontal gyrus (IFG), activity at encoding differed depending on whether items were later recollected regardless of study-test delay. Delay-selective effects were also evident, however. Recollection after $48 \mathrm{~h}$ was associated with enhanced activity in bilateral ventral IFG, whereas recollection after $30 \mathrm{~min}$ was associated with greater fusiform activity. Thus, there is a relationship between the neural activity elicited by an event as it is encoded and the durability of the resulting memory representation.
\end{abstract}

Key words: encoding; memory strength; episodic memory; fMRI; subsequent memory effects; long-term memory

\section{Introduction}

Memories for some events are more durable than others, even when the events engage seemingly equivalent encoding processes. One possibility is that, despite apparent equivalence of study processing, memory durability reflects variation in encoding. Alternatively, memory durability might depend on processes that operate after encoding. For example, memories undergo "synaptic consolidation" for up to several hours (Dudai, 2004; Wixted, 2004). Thus, durability might reflect variation in consolidation.

The present experiment uses the functional magnetic resonance imaging (fMRI) "subsequent memory procedure" to investigate the relationship between encoding and memory durability. Neural activity elicited by study items was classified according to whether or not each item was later remembered. Differences in activity elicited by items that are later remembered versus forgotten ("subsequent memory effects") are interpreted as neural correlates of successful encoding. Several studies have used the procedure to investigate encoding that supports later recollection of words (Wagner et al., 1998; Henson et al., 1999; Davachi et al., 2003; Maril et al., 2003) (for review, see Paller and Wagner, 2002). Subsequent memory effects associated with recollection have consistently been reported in several regions, including left inferior frontal gyrus (IFG), left hippocampus, and left parahippocampal and fusiform cortex.

Received Feb. 24, 2005; revised June 12, 2005; accepted July 3, 2005.

M.R.U. was supported by National Institute of Mental Health National Research Service Award MH14599-26A1. We thank the members of the University of California, Irvine Research Imaging Center for their assistance with fMR data acquisition.

Correspondence should be addressed to Melina R. Uncapher, Center for the Neurobiology of Learning and Memory, University of California, Irvine, Irvine, CA 92697-3800. E-mail: mball@uci.edu. D0I:10.1523/JNEUROSCI.1641-05.2005

Copyright $\odot 2005$ Society for Neuroscience $\quad$ 0270-6474/05/257260-08\$15.00/0
If memory durability is determined solely by factors that operate after encoding, subsequent memory effects will not vary with study-test delay. Under this scenario, the effects index whether study processing was sufficient to establish a memory representation but do not predict its durability. If, however, a study item must be encoded more effectively to be recollected after a long than a short interval, subsequent memory effects will likely vary with delay. For example, longer-term memories might depend on the same encoding operations that support memory over shorter intervals but require these operations to be engaged more extensively. Thus, successful recollection over the short and long term will be associated with common subsequent memory effects, but the effects will be larger for items recollected after the longer interval. Alternatively, additional encoding operations may be required for a memory to survive a long, relative to a short, delay. Under this scenario, subsequent memory effects for longer-term memories will be found in regions beyond those supporting shorter-term memories. Finally, shorter-term memory might be supported by additional operations that impart only a transient benefit. Under this scenario, some subsequent memory effects will be larger for items tested after short than longer delays.

We investigated these scenarios by testing memory after two study-test delays ( 30 min vs $48 \mathrm{~h}$ ). The shorter delay was similar to delays used in most previous subsequent memory experiments (but see Chee et al., 2003; Davachi et al., 2003) [see Stark and Squire (2000) for a study of retrieval effects for delays between 30 min and 1 week]. The longer delay was chosen because it is beyond the period during which synaptic consolidation is thought to occur (Dudai, 2004) and long enough for performance to have declined significantly relative to the shorter delay. The "remem- 
ber $(\mathrm{R}) /$ know $(\mathrm{K})$ " procedure was used to identify items that were recollected after each delay (Yonelinas, 2002).

\section{Materials and Methods}

This study was approved by and performed in accordance with the Institutional Review Board of the University of California, Irvine (UCI).

Participants. Nineteen volunteers [six males; 19-24 years of age; mean, $21.7(\mathrm{SD}=1.6)$ ] gave informed consent to participate in the experiment. All volunteers reported themselves to be right handed and in good general health, with no history of neurological disease or other contraindications for MR imaging, and to have learned English as their first language. Volunteers were recruited from the UCI community and remunerated for their participation. One volunteer's data were excluded because of inadequate subsequent memory performance ( $>2$ SDs below the sample mean at both delays).

Stimulus materials. Critical stimuli were drawn from a pool of 424 concrete words (four to nine letters long; mean written frequency between 1 and 30 counts per million) (Kucera and Francis, 1967). This pool was used to create eight lists of 52 items each, with one-half of the words in each list denoting animate objects and the other half denoting inanimate objects. Study lists of 200 critical items were created from four of the lists, with the extra eight items serving as buffers. Two lists were used to create a pool of 100 new items for the recognition memory test at the 30 min delay (see below, Experimental tasks and procedures), and the remaining two lists were used equivalently for the $48 \mathrm{~h}$ delay. The extra four items at each delay were used as buffers. Word lists were rotated between study and test conditions across subjects. A separate practice study list of eight items was created from the remainder of the word pool.

Experimental tasks and procedures. The experiment consisted of a scanned incidental study phase, followed by two nonscanned recognition memory tests, one administered at $30 \mathrm{~min}$ and the other at $48 \mathrm{~h}$ after the study phase. Instructions and practice for the study phase were given outside the scanner. During the study, volunteers viewed 200 critical words, presented one at a time for $400 \mathrm{~ms}$. They were instructed to decide whether each word represented a living or a nonliving object and to indicate their decision with the index (animate) or middle (inanimate) finger of one hand. The hand used to indicate animacy decisions was counterbalanced across volunteers. Speed and accuracy of responding were given equal emphasis. All words were presented visually in white uppercase letters on a black background centered on fixation. Words subtended maximum horizontal and vertical visual angles of 8 and $1.5^{\circ}$, respectively. During the study phase, words were presented via VisuaStim XGA (Resonance Technology, Northridge, CA) MRI-compatible headmounted display goggles with a field of view of $30^{\circ}$ visual angle and a resolution of $640 \times 480$ pixels.

The 200 critical study items were presented in two blocks, separated by a cued $10 \mathrm{~s}$ break, during one continuous scanning session. One-half of the words in each block were designated for recognition memory test at the 30 min delay (see below), and the remainder was designated for the $48 \mathrm{~h}$ delay. Each block began and ended with two buffer items. Study item stimulus onset asynchrony (SOA) was stochastically distributed with a minimum SOA of $3 \mathrm{~s}$ modulated by the addition of one-third (100) randomly intermixed null trials (Josephs and Henson, 1999). A central fixation cross was present throughout the interitem interval. Study items were presented in pseudorandom order, with no more than three trials of one item type (animate to be tested at $30 \mathrm{~min}$, animate to be tested at $48 \mathrm{~h}$, inanimate to be tested at $30 \mathrm{~min}$, inanimate to be tested at $48 \mathrm{~h}$, or null) occurring consecutively.

Recognition memory was tested outside the scanner for one-half of the study items $30 \mathrm{~min}$ after the conclusion of the study phase and for the remaining half after $48 \mathrm{~h}$. Subjects were not informed that they would undertake a second memory test when they were requested to return to the laboratory after $48 \mathrm{~h}$ (they were merely told that the experiment would be continued at that time and that instructions for the next phase of the experiment would be given after return to the laboratory). Memory was tested using the remember/know procedure (Tulving, 1985), permitting recognized items associated with a phenomenal sense of recollection to be segregated from those associated with an acontextual sense of familiarity (Yonelinas, 2002). The rationale for the use of this procedure was twofold. First, there is accumulating evidence that recognition associated with these two classes of response has dissociable neural bases (Eldridge et al., 2000) (for review, see Yonelinas, 2002). Second, recollection and familiarity have different forgetting rates over retention intervals encompassing those used in the present study, with recollection showing the faster decline (Yonelinas, 2002). Therefore, a method for separating recollection- and familiarity-based recognition is necessary to ensure that contrasts of subsequent memory effects for items tested after $30 \mathrm{~min}$ and $48 \mathrm{~h}$ are not confounded by differential contributions of recollection and familiarity. Specifically, volunteers were instructed to decide whether they recollected seeing the word during the study phase (a remember response), felt that the word was familiar from the previous study phase but could not recollect any contextual information from the study episode (a know response), or judged the word to be new (a new response). The remember/know distinction was explained using standard instructions (Rajaram, 1993), clarified with examples. Responses were made using the index (remember), middle (know), or ring (new) fingers of their right hand. Volunteers were instructed to respond quickly without sacrificing accuracy.

Each test was presented in two consecutive blocks, separated by a short rest period. At each study-test delay, volunteers were presented with 100 old items interspersed among 100 new items, presented one at a time. In each test block, old items consisted of 25 items presented in the first study block and 25 items presented in the second study block (chosen randomly from each study block list). Old and new items were presented pseudorandomly, with no more than three trials of one item type occurring consecutively. Two additional new buffer items were added to the beginning and end of each test block. On each trial, a white fixation cross was presented on a black background for $1000 \mathrm{~ms}$, the screen blanked for $100 \mathrm{~ms}$, and the test word was presented for $300 \mathrm{~ms}$. A response was required during the subsequent $3400 \mathrm{~ms}$ interval, during which the screen remained blanked. Words were presented on a computer monitor with the same display parameters (size, font, and background/text colors) as those used during the study.

fMRI scanning. A Philips Eclipse 1.5T MR scanner (Philips Medical Systems, Andover, MA) was used to acquire both $\mathrm{T}_{1}$-weighted anatomical volume images $\left(256 \times 256\right.$ matrix, $1 \mathrm{~mm}^{3}$ voxels, spoiled gradientrecalled acquisition in a steady state sequence) and $\mathrm{T}_{2}{ }^{*}$-weighted echoplanar images (EPIs) $(64 \times 92$ matrix, $2.6 \times 3.9 \mathrm{~mm}$ pixels, and echo time of $40 \mathrm{~ms}$ ) with blood oxygenation level-dependent (BOLD) contrast. Each EPI volume comprised 27 3-mm-thick axial slices separated by $1.5 \mathrm{~mm}$ and positioned to give full coverage of the cerebrum and most of the cerebellum. Data were acquired in one session comprised of 383 volumes with a repetition time of $2.5 \mathrm{~s} / \mathrm{vol}$. Volumes within sessions were acquired continuously in a descending sequential order. The first four volumes were discarded to allow tissue magnetization to achieve a steady state. The $3.0 \mathrm{~s}$ SOA allowed for an effective sampling rate of the hemodynamic response of $2 \mathrm{~Hz}$.

Data analysis. Data preprocessing and statistical analyses were performed with Statistical Parametric Mapping (SPM2; Wellcome Department of Cognitive Neurology, London, UK; http://www.fil.ion. ucl.ac.uk/spm/) (Friston et al., 1995) implemented in Matlab6 (MathWorks, Natick, MA). All volumes were realigned spatially to the first volume, and the time series for voxels within each slice realigned temporally to the acquisition of the middle slice. Inspection of movement parameters generated during spatial realignment indicated that no volunteer moved $>2 \mathrm{~mm}$ in any direction during any session. Resulting images were spatially normalized to a standard EPI template based on the Montreal Neurological Institute (MNI) reference brain (Cocosco et al., 1997) and resampled into $3 \mathrm{~mm}^{3}$ voxels using nonlinear basis functions (Ashburner and Friston, 1999). Normalized images were smoothed with an isotropic $8 \mathrm{~mm}$ full-width half-maximum Gaussian kernel. Each volunteer's $\mathrm{T}_{1}$ anatomical volume was coregistered to their mean EPI volume and normalized to a standard $\mathrm{T}_{1}$ template of the MNI brain.

Statistical analyses were performed in two stages of a mixed effects model. In the first stage, neural activity was modeled by a $\delta$ function (impulse event) at stimulus onset. These functions were then convolved with a canonical hemodynamic response function (HRF) and its temporal and dispersion derivatives (Friston et al., 1998) to yield regressors in a 
Table 1. Mean (and SD) reaction times (in $\mathrm{ms}$ ) for correctly judged study items as a function of later memory performance at the short and long test delay

\begin{tabular}{llll}
\hline Study-test delay & Remember & Know & Miss \\
\hline $30 \mathrm{~min}$ & $1019(187)$ & $929(135)$ & $953(165)$ \\
$48 \mathrm{~h}$ & $1011(231)$ & $984(162)$ & $949(164)$ \\
\hline
\end{tabular}

general linear model that modeled the BOLD response to each event type. The two derivatives model variance in latency and duration, respectively. Analyses of the parameter estimates pertaining to these derivatives added no theoretically meaningful information to that contributed by the HRF and are not reported (results are available from the corresponding author on request).

Six event types of interest were defined; studied words recollected at 30 $\min (\mathrm{R} 30 \mathrm{~min})$, familiar at $30 \mathrm{~min}(\mathrm{~K} 30 \mathrm{~min})$, or not recognized at 30 $\mathrm{min}$ (M30 min), and studied words recollected at $48 \mathrm{~h}(\mathrm{R} 48 \mathrm{~h})$, familiar at $48 \mathrm{~h}(\mathrm{~K} 48 \mathrm{~h})$, or not recognized at $48 \mathrm{~h}(\mathrm{M} 48 \mathrm{~h})$. Only words that received correct classifications on the study task were included. Words that were incorrectly classified, or for which a response was omitted, were modeled as events of no interest, as were buffer items and the $10 \mathrm{~s}$ rest cue. Six regressors modeling movement-related variance (three rigid-body translations and three rotations determined from the realignment stage) and a session-specific constant term modeling the mean over scans were also used in the design matrix. The time series in each voxel were high-pass filtered to $1 / 128 \mathrm{~Hz}$ to remove low-frequency noise and scaled within session to a grand mean of 100 across both voxels and scans. Parameter estimates for events of interest were estimated using a general linear model. Nonsphericity of the error covariance was accommodated by an $\mathrm{AR}(1)$ model, in which the temporal autocorrelation was estimated by pooling over suprathreshold voxels (Friston et al., 2002). The parameters for each covariate and the hyperparameters governing the error covariance were estimated using restricted maximum likelihood. Effects of interest were tested using linear contrasts of the parameter estimates. These contrasts were carried forward to a second stage in which subjects were treated as a random effect. Only effects surviving an uncorrected threshold of $p<0.001$ and including five or more contiguous voxels were interpreted. The peak voxels of clusters exhibiting reliable effects are reported in MNI coordinates.

Regions of overlap between the outcomes of two contrasts were identified by inclusively masking the relevant SPMs. When the two contrasts were independent, the statistical significance of the resulting SPM was computed using Fisher's method of estimating the conjoint significance of independent tests (Fisher, 1950; Lazar et al., 2002). Unless otherwise specified, each SPM was thresholded at $p<0.01$ to give a conjoint significance of $p<0.001$. Exclusive masking was used to identify voxels where effects were not shared between two contrasts. The SPM constituting the exclusive mask was thresholded at $p<0.05$, whereas the contrast to be masked was thresholded at $p<0.001$. Note that the more liberal the threshold of an exclusive mask, the more conservative is the masking procedure. The hypothesized scenarios outlined in the introduction all relate to the modulation of subsequent memory effects, that is, to effects that take the form of greater activity for study items that were later remembered rather than later forgotten. Accordingly, unidirectional contrasts were used to compare activity elicited by subsequently remembered versus subsequently forgotten items.

\section{Results}

\section{Behavioral performance}

Study task

Animacy decisions for words later tested for recognition memory at $30 \mathrm{~min}$ were made with accuracy equivalent to those later tested at $48 \mathrm{~h}$ [both $93 \%(\mathrm{SD}=3)$ ]. Table 1 lists study reaction times (RTs) conditionalized on later memory performance. To determine whether RT predicted later memory performance as a function of delay, ANOVAs were performed separately for items later accorded remember and know responses. For items associated with later recollection, ANOVA [factors of delay and later performance (remember vs miss)] revealed a main effect of later memory $\left(F_{(1,17)}=16.38 ; p<0.001\right)$, with no interaction between this factor and delay $(F<1)$. Thus, whereas RTs were some $64 \mathrm{~ms}$ longer for items later recollected than those later missed, this difference did not vary according to delay. A second ANOVA, again with factors of delay and later memory classification (know vs miss) revealed a delay-by-memory performance interaction $\left(F_{(1,17)}=7.95 ; p<0.05\right)$. This interaction reflected significantly shorter RTs to study items attracting a subsequent know response at the short delay (929 ms) than those attracting the same response at the long delay (984 ms; $\left.t_{(17)}=3.30 ; p<0.005\right)$. It also reflected significantly longer RTs to study items accorded a know than a new response at the long delay $\left(949 \mathrm{~ms} ; t_{(17)}=2.25 ; p<\right.$ $0.05)$.

\section{Recognition memory}

Recognition memory performance is given in Table 2. Memory performance for old items is based on only those items correctly classified at study. Recollection was estimated by calculating the difference between the probabilities of an $\mathrm{R}$ response to an old versus a new item $\left(p \mathrm{R}_{\text {old }}-p \mathrm{R}_{\text {new }}\right)$. Recollection declined from $0.44(\mathrm{SD}=0.18) 30 \mathrm{~min}$ after study to $0.16(\mathrm{SD}=0.12) 48 \mathrm{~h}$ after study. However, at $48 \mathrm{~h}$, recollection was still significantly greater than zero $\left(t_{(17)}=5.94 ; p<0.0001\right)$. Familiarity was estimated after correcting the proportion of $\mathrm{K}$ responses according to the assumption that recollection and familiarity are independent, such that $p \mathrm{~F}=\left(p \mathrm{~K}_{\text {old }} / 1-p \mathrm{R}_{\text {old }}\right)-\left(p \mathrm{~K}_{\text {new }} / 1-p \mathrm{R}_{\text {new }}\right)$ (Yonelinas and Jacoby, 1995). Familiarity declined from 0.41 ( $\mathrm{SD}=$ $0.18) 30 \mathrm{~min}$ after study, to $0.27(\mathrm{SD}=0.11) 48 \mathrm{~h}$ after study. A two-by-two ANOVA with factors of type of memory (recollection vs familiarity) and delay (30 min vs $48 \mathrm{~h}$ ) revealed a significant effect of delay $\left(F_{(1,17)}=157.06 ; p<0.0001\right)$, and a memory type-by-delay interaction $\left(F_{(1,17)}=7.07 ; p<0.025\right)$. The interaction reflected a greater effect of delay for recollection than familiarity $(0.28$ vs 0.14$)$. However, delay effects were reliable for both types of memory $\left(t_{(17)}=9.22, p<0.0001\right.$ and $t_{(17)}=4.41$, $p<0.0001$ for recollection and familiarity, respectively).

\section{fMRI findings}

We first identified regions in which subsequent memory effects associated with recollection (remember responses) were invariant with respect to delay. We then evaluated the evidence in favor of each of the three scenarios outlined in the Introduction as to how subsequent memory effects might differ according to delay. Accordingly, we sought to identify regions in which (1) subsequent memory effects were common to the two delays but differed in magnitude according to delay and (2) subsequent memory effects were unique to one or the other delay. Analogous contrasts were used to identify delay-invariant and delaysensitive subsequent memory effects associated with familiarity (know responses).

\section{Recollection}

Regions in which subsequent memory effects were invariant with respect to delay were identified by the main effect of subsequent memory (i.e., recollected $>$ forgotten), after exclusive masking by the bidirectional subsequent memory-by-delay interaction (for the outcome of the unmasked contrast, see supplemental Fig. S1, available at www.jneurosci.org as supplemental material). Voxels surviving this procedure are those that demonstrate reliable subsequent memory effects that do not differ significantly $(p>0.05)$ in their magnitude according to delay. As shown in Figure $1 A$ and as listed in Table 3 , regions identified in this anal- 
Table 2. Recognition memory performance (mean and SD) for old and new words at the short and long test delay

\begin{tabular}{|c|c|c|c|c|c|c|}
\hline \multirow[b]{2}{*}{ Study-test delay } & \multicolumn{3}{|l|}{ Old } & \multicolumn{3}{|l|}{ New } \\
\hline & Remember & Know & Miss & Remember false alarm & Know false alarm & Correct rejection \\
\hline $30 \mathrm{~min}$ & $0.51(0.21)$ & $0.30(0.18)$ & $0.18(0.09)$ & $0.07(0.06)$ & $0.18(0.13)$ & $0.74(0.16)$ \\
\hline $48 \mathrm{~h}$ & $0.23(0.15)$ & $0.38(0.13)$ & $0.39(0.13)$ & $0.06(0.06)$ & $0.21(0.10)$ & $0.73(0.12)$ \\
\hline
\end{tabular}
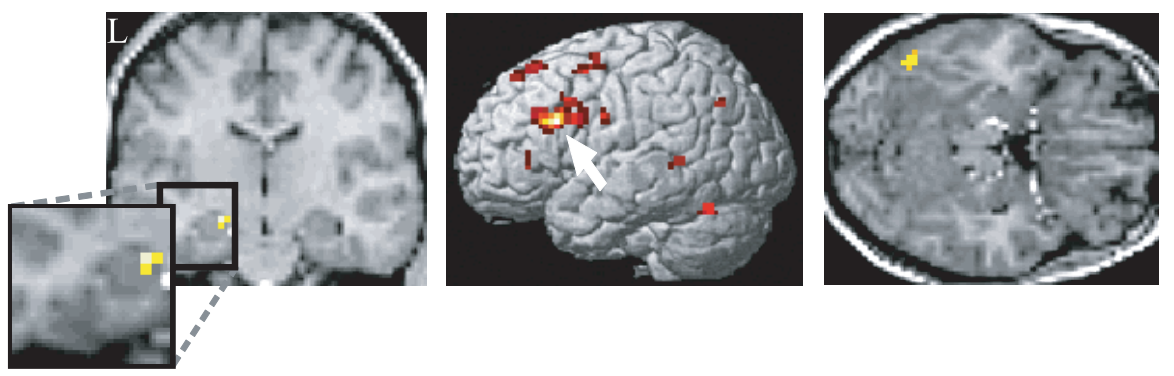

Figure 1. Delay-invariant subsequent memory effects associated with later recollection. Regions in the left anterior hippocampus (left), left IFG (middle), and left fusiform (right) exhibiting subsequent memory effects $(p<0.001)$ that did not vary according to retention interval ( $p>0.05$ ) are shown. Hippocampal and fusiform effects are overlaid onto normalized $\mathrm{T}_{1}$ weighted structural sections of a single randomly chosen volunteer. IFG effects are surface-rendered onto a canonical normalized brain. L, Left hemisphere.

Table 3. Regions showing delay-invariant and delay-selective subsequent memory effects associated with recollection

\begin{tabular}{|c|c|c|c|c|}
\hline & Location $(x, y, z)$ & $\begin{array}{l}\text { Peak Z (number of } \\
\text { voxels) }\end{array}$ & Region & $\begin{array}{l}\text { Approximate } \\
\text { Brodmann area }\end{array}$ \\
\hline \multicolumn{5}{|l|}{ Delay-invariant } \\
\hline & $-18,30,57$ & $3.93(21)$ & L superior frontal gyrus & $6 / 8$ \\
\hline & $0,12,57$ & $3.35(8)$ & Medial superior frontal gyrus & $8 / 6$ \\
\hline & $-45,3,54$ & $3.65(17)$ & L middle frontal gyrus & $6 / 4$ \\
\hline & $-42,18,27$ & $4.62(139)$ & L dorsal inferior frontal gyrus & $9 / 44$ \\
\hline & $-45,33,6$ & $3.37(5)$ & L ventral inferior frontal gyrus & 45 \\
\hline & $57,15,30$ & $3.30(7)$ & R inferior frontal gyrus & 9 \\
\hline & $57,21,15$ & $3.59(9)$ & R inferior frontal gyrus & $45 / 9$ \\
\hline & $39,-6,63$ & $3.83(20)$ & R precentral gyrus & 6 \\
\hline & $-48,-6,30$ & $3.57(9)$ & L precentral gyrus & 6 \\
\hline & $-21,-15,-15$ & $4.23(8)$ & L hippocampus & \\
\hline & $-48,-63,-24$ & $3.92(14)$ & L fusiform & 37 \\
\hline & $-60,-45,3$ & $3.32(10)$ & L superior temporal gyrus & $21 / 22$ \\
\hline & $30,-63,57$ & $4.51(22)$ & R superior parietal & 7 \\
\hline & $-42,-69,36$ & $3.29(5)$ & L medial parietal & 39 \\
\hline \multicolumn{5}{|l|}{ Delay-selective } \\
\hline \multirow[t]{3}{*}{ Long delay } & $-48,39,-3$ & $3.96(13)$ & L ventral inferior frontal gyrus & 47 \\
\hline & $45,45,-6$ & $3.58(7)$ & R ventral inferior frontal gyrus & 47 \\
\hline & $-6,15,54$ & $3.66(5)$ & L superior frontal gyrus & 8 \\
\hline Short delay & $-39,-63,-15$ & $3.44(9)$ & L fusiform gyrus & 37 \\
\hline
\end{tabular}

$Z$ values refer to the peak of the activated cluster. L, Left; $R$, right.

ysis included left anterior hippocampus and dorsal left IFG, regions consistently identified in previous subsequent memory studies (see Introduction). Also identified were left fusiform and medial frontal regions, both of which have also been identified in several previous studies.

We next sought for regions in which subsequent memory effects common to the two delays differed in magnitude according to delay. Common effects were identified by inclusively masking the subsequent memory effects obtained for each delay separately (each thresholded at $p<0.01$, to give a conjoint significance of $p<0.001$ ) (for the outcome of the separate contrasts, see supplemental Fig. S2, available at www.jneurosci.org as supplemental material). To determine whether any of these effects were greater for items tested at the longer delay, this SPM was inclusively masked with the directional interaction between delay and subsequent memory $(\mathrm{R} 48 \mathrm{~h}-\mathrm{M} 48 \mathrm{~h})>(\mathrm{R} 30 \mathrm{~min}-\mathrm{M} 30$ min), also thresholded at $p<0.01$ (see supplemental Fig. S2, available at www. jneurosci.org as supplemental material). No voxels survived the mask. This was also the case when the reverse interaction was used as the mask [i.e., (R30 min - M30 min $)>(\mathrm{R} 48 \mathrm{~h}-\mathrm{M} 48 \mathrm{~h})]$ (see supplemental Fig. S2, available at www.jneurosci.org as supplemental material). Thus, we were unable to identify any region in which subsequent memory effects were common to the two delays yet varied in magnitude according to delay. This remained the case even when the threshold for each of the above three contrasts was reduced to $p<0.05$.

Subsequently, we searched for regions in which subsequent memory effects were uniquely associated with items tested after the 48 h delay. First, we exclusively masked the subsequent memory contrast for this delay with the analogous contrast for the 30 min condition. The outcome of this procedure, which identifies voxels where subsequent memory effects are reliable only for the $48 \mathrm{~h}$ condition, was then inclusively masked with the interaction contrast that identifies where subsequent memory effects are greater at the longer delay $(\mathrm{R} 48 \mathrm{~h}-\mathrm{M} 48 \mathrm{~h})>(\mathrm{R} 30 \mathrm{~min}-$ M30 min), thresholded at $p<0.01$. The outcome of this procedure is an SPM identifying voxels where subsequent memory effects are reliable $(p<0.001)$ for the $48 \mathrm{~h}$ delay only and which, in addition, are significantly greater in magnitude $(p<0.01)$ than at the 30 min delay. [Note that these two contrasts are not redundant. It is entirely possible (and not infrequent) for an effect to be reliable in one condition, but not another, but for the magnitudes of the effects nonetheless not to differ significantly.] Three regions demonstrated the foregoing combination of effects (Fig. 2, Table 3). As shown in the figure, these regions were in bilateral ventral IFG and medial prefrontal cortex. The statistical significance of the subsequent memory-by-delay interaction for each of the peak parameter estimates for each of these regions was determined by ANOVA. The levels of significance for the left and right ventral IFG were $p<0.005$ and $p<0.01$, respectively. For the medial prefrontal region, the interaction was significant at $p<0.01$.

A procedure analogous to that just described was used to identify subsequent memory effects uniquely associated with recol- 

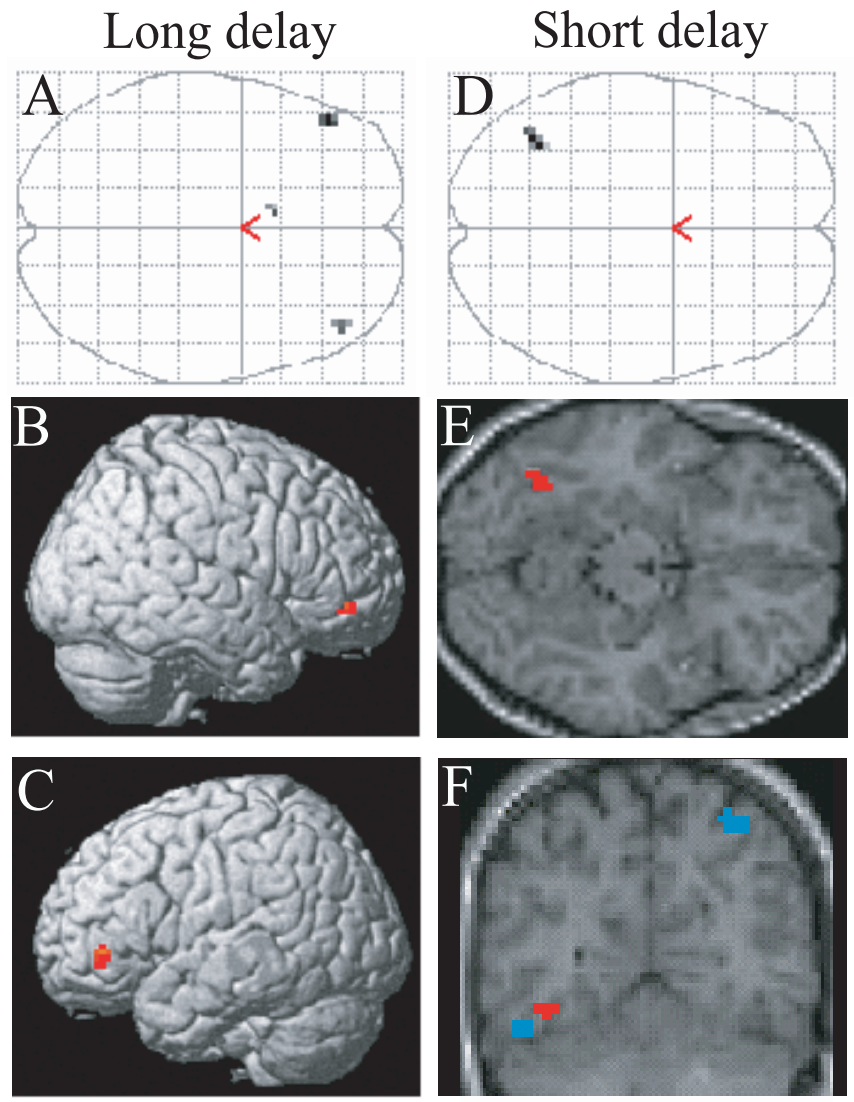

Figure 2. Delay-selective subsequent memory effects associated with later recollection. $\boldsymbol{A}$, Maximum intensity projection illustrating the regional specificity of the subsequent memory effects selective to items recollected after $48 \mathrm{~h}(p<0.001) . B, C$, Surface renderings of these effects in the right $(\boldsymbol{B})$ and left $(\boldsymbol{C})$ ventral IFG. $\boldsymbol{D}$, Maximum intensity projection illustrating the specificity of the subsequent memory effect selective to items recollected after $30 \mathrm{~min}(p<$ $0.001)$. $\boldsymbol{E}$, The same effect overlaid onto an axial section $(z=-15)$ of the $T_{1}$-weighted image from the same volunteer depicted in Figure 1.F, Coronal section $(y=-63)$ illustrating the dissociation between delay-specific (red) and delay-invariant (blue) regions of the left fusiform.

lection after the 30 min delay. As shown in Figure 2, only one region, in left fusiform cortex, exhibited a subsequent memory effect unique to the $30 \mathrm{~min}$ condition. ANOVA of the parameter estimates from the voxel exhibiting the peak subsequent memory effect revealed a subsequent memory-by-delay interaction that was reliable at $p<0.001$.

\section{Familiarity}

Regions demonstrating delay-invariant subsequent memory effects for study items later accorded $K$ judgments were identified by the analogous procedure to that adopted for subsequent recollection effects (see above). Two regions, right parahippocampal gyrus and left precentral gyrus, exhibited such delay-invariant effects (Fig. 3, Table 4).

To investigate the effects of study-test delay on subsequent familiarity, the same procedures were adopted as those used for the analysis of recollection described above. We found no evidence for "subsequent familiarity effects" that were both shared between the two delays but larger for one delay than the other. Nor could we find evidence for effects unique to the long delay. We did, however, identify three regions in which activity was predictive of successful familiarity for the shorter delay only ( $\mathrm{Ta}-$ ble 4). These regions were all located in the precentral gyrus.

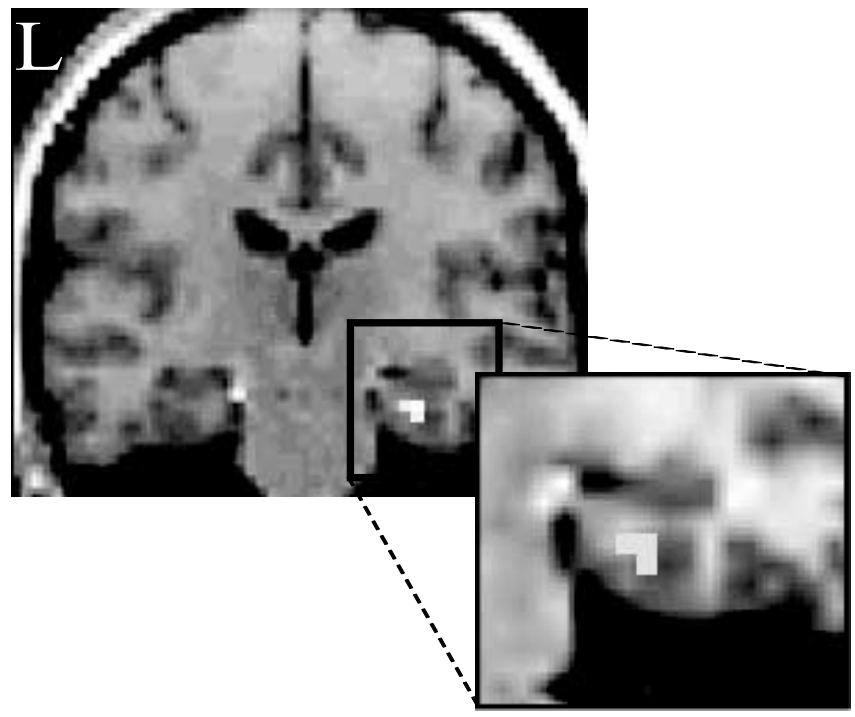

Figure 3. Delay-invariant subsequent memory effects associated with familiarity. The right parahippocampal region in which subsequent memory effects for familiarity $(p<0.001)$ did not differ significantly according to retention interval ( $p>0.05$ ) is shown. Effects are overlaid onto a coronal section $(y=-24)$ of the $T_{1}$-weighted image from the same volunteer as in Figure 1. L, Left hemisphere.

\section{Recollection after 30 min versus familiarity after $48 \mathrm{~h}$}

It has been argued that recognized items associated with remember responses after a short retention interval can "convert" to a know response at longer intervals (Knowlton and Squire, 1995). This implies that, in the present experiment, some items accorded know responses after $48 \mathrm{~h}$ would have been categorized as recollected if they had been tested at $30 \mathrm{~min}$. In turn, this suggests that, for this unknown proportion of items, recollection-based recognition after $30 \mathrm{~min}$ and familiarity-based recognition after $48 \mathrm{~h}$ might be associated with a common pattern of subsequent memory effects. We could find no evidence for such shared effects, however: inclusive masking of the respective subsequent memory effects (each thresholded at $p<0.01$ with a five voxel extent threshold) revealed no overlapping voxels. Although this finding does not preclude the possibility that some items judged familiar after $48 \mathrm{~h}$ might have been recollected if tested at the shorter interval, it does suggest that the two classes of memory judgment depend primarily on independent encoding processes (Davachi and Wagner, 2002; Ranganath et al., 2004).

\section{Discussion \\ Behavioral data}

Consistent with previous behavioral studies using the remember/ know procedure (Gardiner, 1988; Gardiner and Java, 1991; Knowlton and Squire, 1995; Hockley and Consoli, 1999) (for review, see Yonelinas, 2002), recollection declined more than familiarity across delays. On the assumption that any item recollected after $48 \mathrm{~h}$ would have received the same judgment after 30 min, the difference in recollection at the two delays indicates that items recollected after the longer delay were more effectively encoded than the majority $(\sim 60 \%)$ of those recollected after the shorter delay. Applying the same logic to items recognized through familiarity, only $\sim 35 \%$ of items recognized after the long delay were more effectively encoded than those recognized after $30 \mathrm{~min}$. Thus, the power to detect delay-dependent subsequent familiarity effects is less than that for analogous recollection effects.

Study items later recollected were responded to more slowly 
Table 4. Regions showing delay-invariant and delay-selective subsequent memory effects associated with familiarity

\begin{tabular}{cllll}
\hline & Location $(x, y, z)$ & $\begin{array}{l}\text { Peak Z (number of } \\
\text { voxels) }\end{array}$ & Region & $\begin{array}{l}\text { Approximate } \\
\text { Brodmann area }\end{array}$ \\
\hline Delay-invariant & $30,-24,-24$ & $3.76(6)$ & R parahippocampal gyrus & 35 \\
& $-60,-18,45$ & $3.24(5)$ & L precentral gyrus & 6 \\
Delay-selective & & & R precentral gyrus & 4 \\
Short delay & $54,-6,48$ & $3.67(5)$ & R precentral gyrus & 6 \\
& $48,-3,24$ & $3.66(8)$ & L precentral gyrus & 6
\end{tabular}

$Z$ values refer to the peak of the activated cluster. L, Left; $R$, right.

than items later forgotten. Using recognition confidence rather than $\mathrm{R}$ judgments to identify recollected items, Morcom et al. (2003) reported a similar RT difference when using the same study task as that used here. This finding is not invariable, however. Otten and Rugg (2001) reported that study RTs were significantly shorter for items later confidently recognized, whereas in the study by Otten et al. (2001), RTs did not differ according to subsequent memory. Similar fMRI subsequent memory effects were reported in all of these studies, making it unlikely that the effects merely reflect differential study RTs (Wagner et al., 1998). Crucially, in the present experiment, there was no interaction between subsequent recollection effects on study RT and studytest delay. Thus, interactions between delay and fMRI subsequent recollection effects cannot be attributed to differential study performance. This is not true for familiarity-based recognition, however. In this case, longer-study RTs for later recognized relative to forgotten items were found after $48 \mathrm{~h}$ only.

\section{fMRI data}

Recollection

Delay-invariant subsequent memory effects were found in several regions identified in previous studies, including left hippocampus, left IFG, and left fusiform cortex (Otten et al., 2001; Morcom et al., 2003). By themselves, these effects might suggest that memory durability is determined solely by factors operating after encoding. However, the finding of additional subsequent memory effects that did vary with delay is inconsistent with this proposal. These delay-specific effects demonstrated a regional double dissociation, suggesting that recollection after the short and the long delays benefited from functionally distinct encoding operations. Thus, the findings indicate that even when study conditions are controlled, there is sufficient variability in how items are encoded to influence the durability of the resulting memory representation. Of course, these findings do not imply that memory durability does not also depend on processes operating after encoding. They do, however, suggest that postencoding processes are not so potent as to override the influence of variability in encoding operations.

The subsequent memory effects selective for items recollected after $30 \mathrm{~min}$ were localized to left fusiform cortex, whereas those selective for the $48 \mathrm{~h}$ condition were in bilateral ventral IFG. The peak coordinates of the fusiform region correspond with the "visual word form area," a region considered specialized for the processing of orthography (McCandliss et al., 2003; Hillis et al., 2005). Thus, it would appear that recollection of study items that received relatively greater orthographic processing was facilitated, but only transiently. In contrast, the ventral IFG region that exhibited subsequent recollection effects for the $48 \mathrm{~h}$ delay corresponded to regions identified by numerous authors as supporting one or more aspects of semantic processing (Bookheimer, 2002; Buckner and Koutstaal, 1998; Gabrieli et al., 1998; Poldrack et al., 1999; Wagner et al., 1998, 1999, 2001). Strikingly, there is almost perfect overlap with the bilateral ventral IFG regions identified by Shivde and ThompsonSchill (2004) as supporting maintenance within semantic working memory. Thus, it appears that recollection after $48 \mathrm{~h}$ was facilitated by the engagement during encoding of semantic processes additional to those required to support memory after 30 min, when memory representations based on other stimulus attributes such as the orthographic structure of an item were presumably still accessible. Implicit in this proposal is the possibility that, in addition to influencing the durability of the resulting memory representations, the encoding operations reflected in delay-specific subsequent memory effects also influenced the content of what was recollected at each delay. Thus, the present findings fit with behavioral evidence that memory for surface details of an event declines rapidly over time, leaving a more general, semantically mediated representation of its "gist" (for review, see Koriat et al., 2000).

The delay-specificity of the fusiform and ventral IFG effects discussed above may reflect the combined influences of the differential longevity of different kinds of encoded information and variation in the way recognition test items were processed at each delay. Specifically, subjects may have adopted different "retrieval orientations" (Rugg and Wilding, 2000; Dobbins et al., 2003) when performing the recognition memory tests after each delay. At the shorter interval, the processing of test items may have emphasized relatively superficial, perceptual features at the expense of semantic information. This retrieval strategy would have benefited items for which orthographic processing had been emphasized at study. The transience of such representations would, however, render this retrieval strategy ineffectual at the longer delay. When tested after $48 \mathrm{~h}$, subjects may have adopted a retrieval orientation that emphasized more general, semantic features of the test items, leading to a bias in favor of the retrieval of items that received relatively extensive semantic processing during study.

What is the relationship between the delay-selective subsequent memory effects just discussed and the delay-invariant effects discussed previously? We turn first to the hippocampus. The role of the hippocampus in encoding is generally held to be the integration ("binding") of disparate cortical inputs into a cohesive memory representation (Squire and Zola-Morgan, 1991; Eichenbaum, 2000; O'Reilly and Rudy, 2001). One hypothesis is that hippocampal subsequent memory effects are a reflection of the relatively greater cortical activity elicited by later recollected items (Otten et al., 2001) [for a similar view, see Wagner et al. (1998)]. Thus, the magnitude of hippocampal subsequent memory effects will vary with the amount of cortically derived information to be integrated into an episodic representation but not with its content. In the present experiment, items recollected after the two delays were each associated with cortical subsequent memory effects in addition to the delay-independent effects. The net cortical input to the hippocampus during the successful encoding of items recollected after each delay may therefore have been similar, giving rise to similar hippocampal subsequent memory effects.

At the cortical level, we suggest that the encoding operations reflected in the delay-selective and delay-invariant subsequent memory effects act in a synergistic manner. By this argument, the 
delay-invariant effects reflect "core" encoding operations, without which an episodic memory representation is unlikely to be formed. The delay-selective effects reflect processing that further emphasizes specific stimulus attributes that are differentially beneficial for memory tested after each delay. This does not necessarily mean, however, that the additional processing reflected in delay-selective subsequent memory effects is qualitatively different from that reflected by the delay-invariant effects. As is evident in Figure 2, the subsequent memory effect selective for the short delay is localized adjacent to the fusiform region in which effects were delay selective. Similarly, the ventral IFG region, demonstrating effects for items tested after the long delay, has been argued to be functionally contiguous with dorsal/posterior IFG (Gold and Buckner, 2002; Gold et al., 2005), in which, in the present study, effects were delay invariant. It is therefore possible that delay-selective effects reflect recruitment of additional neuronal resources to encoding operations that are also reflected by delay-invariant effects rather than engagement of qualitatively distinct operations.

\section{Familiarity}

A delay-invariant subsequent familiarity effect was found in right parahippocampal cortex, consistent with other reports of such effects in extrahippocampal medial temporal cortex (Davachi et al., 2003; Ranganath et al., 2004). Regions were also identified in which subsequent familiarity effects varied with study-test delay. These were in frontal areas associated with motor functions, raising the possibility that they are correlates of the delay-selective RT differences associated with items accorded know responses.

\section{Conclusions}

The present findings suggest that the durability of an episodic memory representation is at least partly determined by variation in neural activity engaged at encoding. This variability does not occur within a single "encoding circuit." Rather, the encoding of episodic memory representations capable of surviving a retention interval of days, rather than minutes, involves recruitment of additional neural resources that likely support more extensive semantic processing of the study item. Recollection of items after a relatively short interval is also associated with delay-specific encoding-related activity. In this case, however, the activity reflects greater processing of low-level nonsemantic attributes of the study item. Together, these findings suggest a neural basis for the observation that the decline in memory performance as retention interval increases is accompanied by a decline in memory for specific details. They underscore the fact that, as retention interval increases, memory representations supporting performance not only differ in a quantitative sense (e.g., by losing "strength") but also qualitatively.

\section{References}

Ashburner J, Friston KJ (1999) Nonlinear spatial normalization using basis functions. Hum Brain Mapp 7:254-266.

Bookheimer S (2002) Functional MRI of language: new approaches to understanding the cortical organization of semantic processing. Annu Rev Neurosci 25:151-188.

Buckner RL, Koutstaal W (1998) Functional neuroimaging studies of encoding, priming, and explicit memory retrieval. Proc Natl Acad Sci USA 95:891-898.

Chee MW, Westphal C, Goh J, Graham S, Song AW (2003) Word frequency and subsequent memory effects studied using event-related fMRI. NeuroImage 20:1042-1051.

Cocosco C, Kollokian V, Kwan RS, Evans A (1997) Brainweb: online interface to a 3D MRI simulated brain database. NeuroImage 5:S425.

Davachi L, Wagner AD (2002) Hippocampal contributions to episodic en- coding: insights from relational and item-based learning. J Neurophysiol 88:982-990.

Davachi L, Mitchell JP, Wagner AD (2003) Multiple routes to memory: distinct medial temporal lobe processes build item and source memories. Proc Natl Acad Sci USA 100:2157-2162.

Dobbins IG, Rice HJ, Wagner AD, Schacter DL (2003) Memory orientation and success: separable neurocognitive components underlying episodic recognition. Neuropsychologia 41:318-333.

Dudai Y (2004) The neurobiology of consolidations, or, how stable is the engram? Annu Rev Psychol 55:51-86.

Eichenbaum H (2000) A cortical-hippocampal system for declarative memory. Nat Rev Neurosci 1:41-50.

Eldridge LL, Knowlton BJ, Furmanski CS, Bookheimer SY, Engel SA (2000) Remembering episodes: a selective role for the hippocampus during retrieval. Nat Neurosci 3:1149-1152.

Fisher RA (1950) Statistical methods for research workers, Ed 11. London: Oliver and Boyd.

Friston KJ, Holmes AP, Worsley KJ, Poline JB, Frith CD, Frackowiak RSJ (1995) Statistical parametric maps in functional imaging: a general linear approach. Hum Brain Mapp 189-210.

Friston KJ, Fletcher P, Josephs O, Holmes A, Rugg MD, Turner R (1998) Event-related fMRI: characterizing differential responses. NeuroImage 7:30-40.

Friston KJ, Penny W, Phillips C, Kiebel S, Hinton G, Ashburner J (2002) Classical and Bayesian inference in neuroimaging: theory. NeuroImage 16:465-483.

Gabrieli JD, Poldrack RA, Desmond JE (1998) The role of left prefrontal cortex in language and memory. Proc Natl Acad Sci USA 95:906-913.

Gardiner JM (1988) Functional aspects of recollective experience. Mem Cognit 16:309-313.

Gardiner JM, Java RI (1991) Forgetting in recognition memory with and without recollective experience. Mem Cognit 19:617-623.

Gold BT, Buckner RL (2002) Common prefrontal regions coactivate with dissociable posterior regions during controlled semantic and phonological tasks. Neuron 35:803-812.

Gold BT, Balota DA, Kirchhoff BA, Buckner RL (2005) Common and dissociable activation patterns associated with controlled semantic and phonological processing: evidence from fMRI adaptation. Cereb Cortex, in press.

Henson RN, Rugg MD, Shallice T, Josephs O, Dolan RJ (1999) Recollection and familiarity in recognition memory: an event-related functional magnetic resonance imaging study. J Neurosci 19:3962-3972.

Hillis AE, Newhart M, Heidler J, Barker P, Herskovits E, Degaonkar M (2005) The roles of the "visual word form area" in reading. NeuroImage 24:548-559.

Hockley WE, Consoli A (1999) Familiarity and recollection in item and associative recognition. Mem Cognit 27:657-664.

Josephs O, Henson RN (1999) Event-related functional magnetic resonance imaging: modelling, inference and optimization. Philos Trans R Soc Lond B Biol Sci 354:1215-1228.

Knowlton BJ, Squire LR (1995) Remembering and knowing: two different expressions of declarative memory. J Exp Psychol Learn Mem Cogn 21:699-710.

Koriat A, Goldsmith M, Pansky A (2000) Toward a psychology of memory accuracy. Annu Rev Psychol 51:481-537.

Kucera H, Francis W (1967) Computational analysis of present-day American English. Providence, RI: Brown UP.

Lazar NA, Luna B, Sweeney JA, Eddy WF (2002) Combining brains: a survey of methods for statistical pooling of information. NeuroImage 16:538-550.

Maril A, Simons JS, Mitchell JP, Schwartz BL, Schacter DL (2003) Feelingof-knowing in episodic memory: an event-related fMRI study. NeuroImage 18:827-836.

McCandliss BD, Cohen L, Dehaene S (2003) The visual word form area: expertise for reading in the fusiform gyrus. Trends Cogn Sci 7:293-299.

Morcom AM, Good CD, Frackowiak RS, Rugg MD (2003) Age effects on the neural correlates of successful memory encoding. Brain 126:213-229.

O’Reilly RC, Rudy JW (2001) Conjunctive representations in learning and memory: principles of cortical and hippocampal function. Psychol Rev 108:311-345.

Otten LJ, Rugg MD (2001) Task-dependency of the neural correlates of episodic encoding as measured by fMRI. Cereb Cortex 11:1150-1160. 
Otten LJ, Henson RN, Rugg MD (2001) Depth of processing effects on neural correlates of memory encoding: relationship between findings from across- and within-task comparisons. Brain 124:399-412.

Paller KA, Wagner AD (2002) Observing the transformation of experience into memory. Trends Cogn Sci 93-102.

Poldrack RA, Wagner AD, Prull MW, Desmond JE, Glover GH, Gabrieli JD (1999) Functional specialization for semantic and phonological processing in the left inferior prefrontal cortex. NeuroImage 10:15-35.

Rajaram S (1993) Remembering and knowing: two means of access to the personal past. Mem Cognit 21:89-102.

Ranganath C, Yonelinas AP, Cohen MX, Dy CJ, Tom SM, D’Esposito M (2004) Dissociable correlates of recollection and familiarity within the medial temporal lobes. Neuropsychologia 42:2-13.

Rugg MD, Wilding EL (2000) Retrieval processing and episodic memory. Trends Cogn Sci 4:108-115.

Shivde G, Thompson-Schill SL (2004) Dissociating semantic and phonological maintenance using fMRI. Cogn Affect Behav Neurosci 4:10-19.

Squire LR, Zola-Morgan S (1991) The medial temporal lobe memory system. Science 253:1380-1386.

Stark CE, Squire LR (2000) fMRI activity in the medial temporal lobe during recognition memory as a function of study-test interval. Hippocampus $10: 329-337$.

Tulving E (1985) Memory and consciousness. Can Psychol 26:1-12.

Wagner AD, Schacter DL, Rotte M, Koutstaal W, Maril A, Dale AM, Rosen BR, Buckner RL (1998) Building memories: remembering and forgetting of verbal experiences as predicted by brain activity. Science 281:1188-1191.

Wagner AD, Koutstaal W, Schacter DL (1999) When encoding yields remembering: insights from event-related neuroimaging. Philos Trans R Soc Lond B Biol Sci 354:1307-1324.

Wagner AD, Pare-Blagoev EJ, Clark J, Poldrack RA (2001) Recovering meaning: left prefrontal cortex guides controlled semantic retrieval. Neuron 31:329-338.

Wixted JT (2004) The psychology and neuroscience of forgetting. Annu Rev Psychol 55:235-269.

Yonelinas AP (2002) The nature of recollection and familiarity: a review of 30 years of research. J Mem Lang 441-517.

Yonelinas AP, Jacoby LL (1995) Dissociating automatic and controlled processes in a memory-search task: beyond implicit memory. Psychol Res $57: 156-165$. 